\section{Identificación de factor de crecimiento vascular endotelial en células glandulares de tejido prostático maligno y benigno. Relación con la recidiva tumoral al año de la prostatectomía}

\author{
PEDRO ACUÑA ${ }^{1}$, ANDRÉS ELLWANGER $^{\mathrm{a}}$, \\ ANGELA RAMÍREZ ${ }^{\mathrm{a}}$, FELIPE CARDEMIL ${ }^{5}$, \\ JORGE VEGA ${ }^{3}$, RENATO CASALINO ${ }^{4}$, EVA MADRID ${ }^{2,5}$
}

\section{Vascular endothelial growth factor in malignant and non malignant prostatic tissue. Association with tumor recurrence at one year after prostatectomy}

Background: Prostate cancer (PC) is the second cause of death by cancer in men in Chile. Its behavior is so variable that it is necessary to search reliable prognostic markers. Vascular Endothelial Growth Factor (VEGF) is one of the most powerful proangiogenic factors. There is no agreement on its validity as a diagnostic or prognostic factor. Aim: To search for VEFG in prostatic tissue. Material and Methods: This study was performed in prostatectomy tissue coming from 41 patients with PC and 39 patients with benign prostatic hyperplasia (BPH). Specimens were studied using immunohistochemical staining for VEGF. The percentage of stained glandular cells per patient was calculated and associated with pathological diagnosis in cancer patients. Results: PC biopsies had a mean of $82 \%$ of VEGF (+) stained cells, while BPH had only $1.6 \%(p<0.01)$. No relationship was found between the percentage of staining and recurrence at one year of follow-up in the case of $P C$. Conclusions: These results would rule out VEGF as a prognostic factor in this series of patients.

(Rev Med Chile 2013; 141: 153-159).

Key words: Prostatic hyperplasia; Prostatic neoplasms; Prostate-specific antigen; Vascular endothelial growth factors.

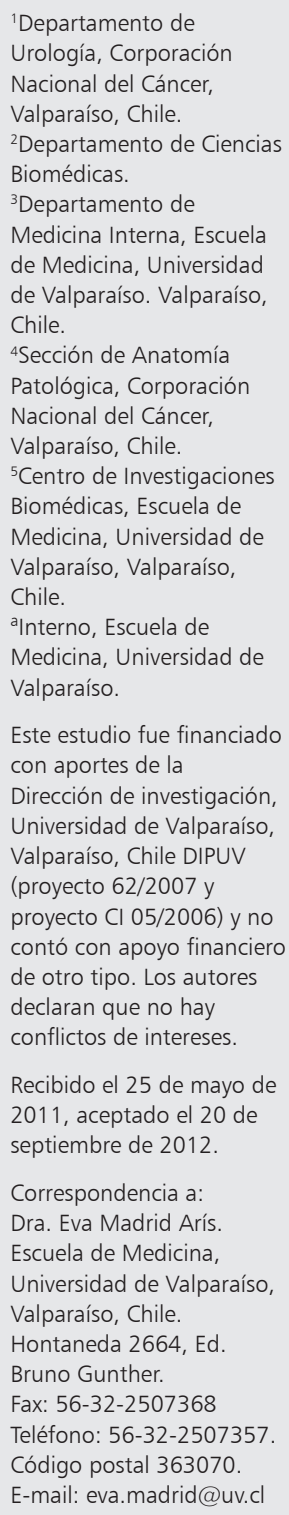

E l cáncer de próstata $(\mathrm{CaP})$ es la segunda causa de muerte por cáncer en hombres en Chile. La tasa de mortalidad se ha duplicado en los últimos 20 años, aumentando su cifra de 10,8 por 100.000 hombres en 1990 a 20,2 en $2008^{1,2}$, a pesar de la implementación rutinaria de la determinación del antígeno prostático específico (PSA) como prueba diagnóstica. Su historia natural es tan variada e impredecible que ha llevado a la búsqueda permanente de factores pronósticos ${ }^{3}$; los utilizados actualmente son el índice de Gleason y la medición del antígeno prostático específico (PSA). Aun así, estos factores tienen bajo valor pronóstico en las neoplasias que se diagnostican actualmente, las que al momento del diagnóstico tienen un índice de Gleason $\leq 6$ y un PSA menor a $10 \mathrm{ng} / \mathrm{ml}^{4}$. Se espera que en los países desarrollados, más de $80 \%$ de los $\mathrm{CaP}$ se diagnostiquen con PSA $<10 \mathrm{ng} / \mathrm{ml}$, por tanto, se hace necesario encontrar otros marcadores que se relacionen con el pronóstico del tumor ${ }^{5}$. 
Uno de los potenciales factores pronósticos que se está estudiando ampliamente es el factor de crecimiento vascular endotelial (VEGF), por su relación, no sólo con la angiogénesis tumoral, sino también con otros fenómenos descritos más recientemente, como la transición epitelio-mesenquimática (EMT), inhibición de la apoptosis, capacidad migratoria de las células, capacidad adhesiva a la matriz y proliferación de las células tumorales por vías autocrinas y paracrinas ${ }^{6-13}$. El concepto de EMT en oncología es relativamente nuevo y podría reflejar un proceso de transdiferenciación en vez de un proceso de desdiferenciación ${ }^{15}$. La EMT genera células de distinto fenotipo y función, movilidad celular, capacidad de invasión del tejido local y permite inestabilidad genética en las células cancerosas para adquirir un fenotipo mesenquimático y facilitar una diseminación local y sistémica ${ }^{6,14-15}$. En los últimos años se ha ido demostrando una clara relación entre los niveles de VEGF y la EMT de las células tumorales, por lo que la inhibición de VEGF mediante anticuerpos neutralizantes podría producir inhibición del crecimiento del tumor y de la capacidad metastatizante de sus células ${ }^{11}$.

La presencia de diferentes patrones de tinción para VEGF entre células prostáticas malignas y normales es controversial. Algunos autores sugieren que VEGF está presente en células prostáticas malignas y no en hiperplasia prostática o células normales ${ }^{16,17}$, mientras que otros autores comunican que no hay diferencia ${ }^{18,19}$. No es claro si estas contradicciones están relacionadas con la etnia, factores locales, con la técnica de recuperación antigénica durante la inmunohistoquímica o con fallas de diseño metodológico de los trabajos (ya que la mayoría ha trabajado con números muestrales pequeños y técnica de muestreo no explicitado). La relación entre la expresión de VEGF y el pronóstico del cáncer luego de una prostatectomía radical no ha sido determinada.

Debido a la diversidad de resultados en los estudios previos, es necesario continuar con la investigación sobre la presencia de VEGF en el cáncer prostático y su eventual asociación con los marcadores en uso actualmente en Chile, como los niveles de PSA preoperatorio y el índice de Gleason.

El objetivo del presente estudio fue determinar si existe diferencia entre la presencia de VEGF en el tejido glandular prostático maligno y no maligno en pacientes chilenos; $y$ si VEGF se comporta como predictor de una recidiva de la enfermedad al año de seguimiento, comparándola con los factores pronósticos utilizados en la actualidad.

\section{Material y Métodos}

\section{Pacientes}

Se realizó un estudio de casos y controles en los laboratorios del Centro de Investigaciones Biomédicas de la Escuela de Medicina de la Universidad de Valparaíso. Se reclutaron todos los pacientes diagnosticados de CaP entre los años 2002 y 2008 en la Corporación Nacional del Cáncer de Valparaíso CONAC, y un grupo control de pacientes con HPB sometidos a prostatectomía. Se registró la información demográfica, clínica y de laboratorio de cada paciente, índice de Gleason en el tejido extraído en la prostatectomía, PSA preoperatorio y a 12 meses de la cirugía. Los criterios de inclusión fueron: $\mathrm{CaP}$ recién diagnosticado con indicación de prostatectomía exclusiva o HPB diagnosticada por biopsia. Los pacientes con compromiso ganglionar, márgenes quirúrgicos positivos en la biopsia, metástasis a distancia, tratamiento hormonal previo o radioterapia, fueron excluidos. Se obtuvo un número de pacientes con cáncer de 41 y el grupo control de 39 pacientes. El Comité de Ética de la Facultad de Medicina de la Universidad de Valparaíso aprobó la realización de este estudio. Los pacientes con $\mathrm{CaP}$ se dividieron en dos grupos según la presencia o no de recidiva bioquímica, la cual se definió como un PSA al año de la prostatectomía mayor de $0,2 \mathrm{ng} / \mathrm{ml}$.

\section{Immunohistoquímica}

Las biopsias fueron fijadas en formalina al 10\% neutralizada con carbonato de calcio a saturación, y luego incluidas en tacos de Paraplast. Los tacos fueron remitidos por el patólogo de la CONAC a los laboratorios de la Universidad de Valparaíso, donde se procesaron. Se cortaron láminas de $5 \mu \mathrm{m}$ de grosor para el estudio inmunohistoquímico. Se desparafinó la muestra, las láminas fueron tratadas con $\mathrm{H}_{2} \mathrm{O}_{2}$ al 3\% (v/v) en PBS por 30 min para bloquear la actividad de la peroxidasa endógena. Cada uno de los siguientes pasos se siguió con un enjuague con PBS. Todos los pasos fueron realizados en una cámara húmeda, y se puso cuidado en evitar la desecación de las muestras. La recuperación antigénica fue realizada por inmersión de la lámina en buffer (TRIS-HCl $10 \mathrm{mM}, \mathrm{pH}$ 10) por $30 \mathrm{~min}$ a $95^{\circ} \mathrm{C}$. El marcaje no específico 
fue bloqueado incubando la lámina con solución de Cas-Block (Zymed Laboratories, South San Francisco, CA, USA) por 10 min. Los cortes fueron incubados a $4^{\circ} \mathrm{C}$ durante la noche, con el anticuerpo policlonal anti humano VEGF hecho en conejo (VEGF-A, VEGF-B, VEGF-C, VEGF-D, LabVision, USA), diluido en 1:500 en PSB Tween 20 al 0,3\% (v/v). Luego de enjuagar con PBS, todos los cortes fueron incubados por $1 \mathrm{~h}$ a temperatura ambiente con IgG de cabra anti conejo conjugado con biotina (Rockland, Gilbertsville, PA, USA), diluido en PBS a 1:1.000. Luego del enjuague en PBS, los cortes fueron incubados con el kit Vectastain $\mathrm{ABC}$ (Vector) por $1 \mathrm{~h}$ a temperatura ambiente. La reacción de peroxidasa se visualizó usando el kit NovaRED (Vector, Burlingame, CA, USA). Luego de la inmunomarcación, se hizo contratinción con hematoxilina de Meyer (Merck, Darmstadt, Alemania). Para cada reacción inmunohistoquímica, se realizaron controles negativos que fueron incubados con suero de conejo normal u omitiendo el anticuerpo primario. Se usó cortes de angiosarcoma como control positivo.

\section{Análisis de Imágenes}

Las muestras fueron fotografiadas mediante el microscopio Inverted System Microscope Olympus IX81 con Cámara Olympus CP71, con un aumento de 60x. Se seleccionaron 5 campos por paciente elegidos al azar y se analizaron mediante el programa Image Pro Plus. El programa permite la cuantificación manual de las células glandulares, diferenciando si están positivas o negativas para VEGF según su coloración: siendo positivas las células cuyo citoplasma adquirió un color rojo y negativas si mantuvieron el color morado claro de la hematoxilina. El conteo de células se realizó por 4 observadores distintos, no patólogos, que no estaban al tanto del diagnóstico de cada paciente. Se obtuvo el total de células marcadas y células totales de las 5 imágenes y se calculó el porcentaje de marcaje por paciente.

\section{Análisis estadístico}

Las variables continuas se describieron como promedios y desviaciones estándar en caso de ser de distribución normal, y como mediana y rango intercuartil si esta condición no se cumplía; las variables categóricas, por medio de frecuencias absolutas y relativas. Se usó la prueba de Shapiro Wilk para definir el tipo de distribución. Se usó la prueba de $t$ de Student para comparar variables continuas cuando la distribución fue normal, y Mann Whitney cuando la distribución no lo fue. Para evaluar correlación entre variable cuantitativas se uso la prueba de Kendall. Para la significancia estadística se determinó un $\mathrm{p}<0,01$.

Se usó el programa Stata 10.0 para el análisis estadístico.

Este manuscrito contiene información publicada preliminarmente en la Revista Chilena de Urología ${ }^{20}$; la republicación de los datos basales se hace con el conocimiento y aprobación de los editores de la Revista Chilena de Urología y de la Revista Médica de Chile.

\section{Resultados}

La información de los pacientes al momento de la prostatectomía, índice de Gleason y antígeno prostático específico en cada grupo se muestra en Tabla 1.

Tabla 1. Presencia de factor de crecimiento vascular endotelial (VEGF) en células de hiperplasia prostática benigna (HPB) y cáncer prostático

\begin{tabular}{|lccc|}
\hline Variable & HPB & Cáncer & Valor de $\mathbf{p}^{*}$ \\
\hline Edad (años) & $\mathbf{n}=\mathbf{3 9}$ & $\mathbf{n}=\mathbf{4 1}$ & 0,0002 \\
Índice de Gleason (puntos) & $70,7 \pm 7,37$ & $64,6 \pm 6,75$ & 0,0163 \\
PSA preoperatorio (ng/ml) & - & $6^{* *}$ & \\
PSA post-operatorio (12 meses) (ng/dl) & $4,52 \pm 2,36$ & $18,7 \pm 40,7$ & $<0,0001$ \\
\hline Porcentaje de marcaje & -- & $2,19 \pm 11,5$ & $82,1 \pm 17,6$ \\
\hline
\end{tabular}

Valores expresados en promedios y desviaciones standard. HPB: Hiperplasia Prostática Benigna; PSA: Antígeno Prostático Específico. ${ }^{*}$ Prueba $t$ de Student. ${ }^{* *}$ moda. 
Luego de analizar 5 campos microscópicos por paciente, las pruebas de hipótesis mostraron una diferencia significativa entre el marcaje de las células glandulares neoplásicas e hiperplásicas que fue de $82,1 \pm 17,6 \%$ y $1,56 \pm 1,93 \%$, respectivamente $(\mathrm{p}<0,0001)$. El marcaje se muestra en las Figuras 1 y 2 .
La correlación de Kendall se calculó entre porcentaje de marcaje para VEGF e índice de Gleason en los pacientes con cáncer y no se encontró una correlación significativa (tau $=-0,17 ; \mathrm{p}=0,16)$.

También se calculó la correlación de Kendall entre porcentaje de marcaje para VEGF y nivel de antígeno prostático específico preoperatorio en
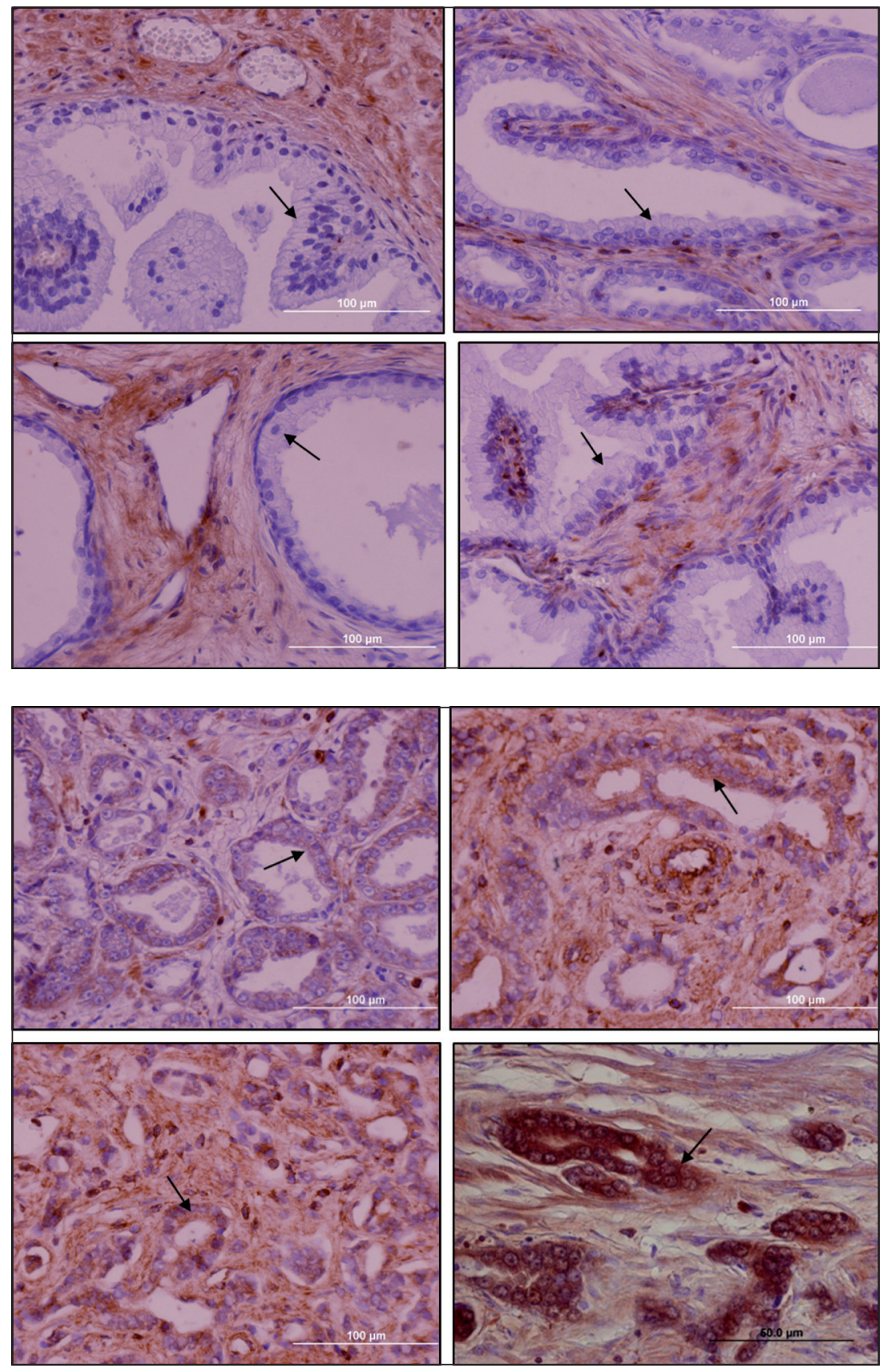

Figura 1. Inmunomarcación para factor de crecimiento endotelial vascular (VEGF) en tejido prostático benigno. Se presentan cortes de próstatas con Hiperplasia Benigna. Se observa que el citoplasma de las células glandulares prostáticas (flechas) no tienen marcación para VEGF (mantiene la coloración propia de la tinción con Hematoxilina-Eosina).

Figura 2. Inmunomarcación para factor de crecimiento endotelial vascular (VEGF) en tejido prostático maligno. Se presentan cortes de Cáncer de Próstata. Se observa que el citoplasma de las células glandulares prostáticas (flechas) tienen marcación positiva para VEGF (adquieren coloración roja). 


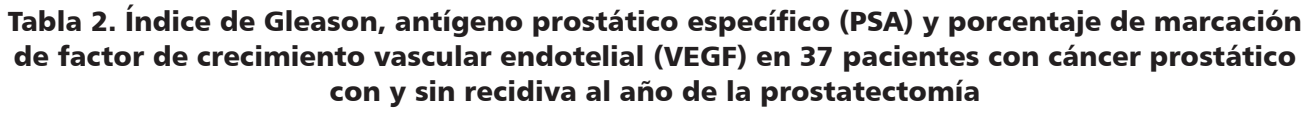

\begin{tabular}{|c|c|c|c|c|c|}
\hline \multirow[b]{2}{*}{ Edad } & \multicolumn{2}{|c|}{$\begin{array}{l}\text { Recidiva negativa } \\
\begin{array}{c}\text { (PSA } \leq 0,2 \mathrm{ng} / \mathrm{ml}) \\
\mathrm{n}=21\end{array}\end{array}$} & \multicolumn{2}{|c|}{$\begin{array}{c}\text { Recidiva positiva } \\
\begin{array}{c}\text { (PSA }>0,2 \mathrm{ng} / \mathrm{ml}) \\
n=16\end{array}\end{array}$} & \multirow{2}{*}{$\begin{array}{c}\text { Valor de } \boldsymbol{p} \text { * } \\
\text { NS }\end{array}$} \\
\hline & 63 & $(60-69)$ & 65 & $(61-69,5)$ & \\
\hline Índice de Gleason (puntos) & 6 & $(5,5-7)$ & 6,5 & $(6-7)$ & NS \\
\hline PSA pre-operatorio (ng/ml) & 7,0 & $(6,0-8,5)$ & 7,25 & $(5,9-12,5)$ & NS \\
\hline PSA al año postoperatorio & \multicolumn{2}{|c|}{$0,002(0,001-0,015)$} & 0,4 & $(0,29-0,8)$ & $<0,0001$ \\
\hline Porcentaje de marcación & 86,6 & $(72,0-93,0)$ & 89,55 & $(77,0-92,3)$ & NS \\
\hline
\end{tabular}

PSA: Antígeno prostático específico; VEGF: Factor de crecimiento vascular endotelial. *Mann Whitney U. ** Valores expresados en medianas y rango intercuartílico.

los pacientes con cáncer, no encontrándose una correlación significativa ( $\operatorname{tau}=0,07 ; \mathrm{p}=0,53$ ).

Los pacientes con diagnóstico de CaP se dividieron según el valor de su PSA de control al año en dos grupos (recidiva vs no recidiva). La distribución de los datos por grupo se muestra en la Tabla 2. El porcentaje de marcaje no mostró relación con la recidiva $(\mathrm{p}=0,157)$. Tampoco el PSA preoperatorio y el índice de Gleason mostraron relación con la recidiva bioquímica al año de seguimiento ( $\mathrm{p}=0,155$ y $\mathrm{p}=0,085$ respectivamente).

\section{Discusión}

Este estudio mostró diferencias en la presencia de VEGF en las células glandulares de las biopsias prostáticas de los pacientes con CaP y HPB. Este hallazgo puede estar relacionado no sólo con angiogénesis inducida por hipoxia, sino que también con la transición de células secretoras de un fenotipo epitelial a uno mesenquimático y con el reclutamiento de células quiescientes al ciclo celular ${ }^{10}$.

El patrón de marcaje de VEGF es controversial como factor pronóstico para la evolución del $\mathrm{CaP}^{21-25}$, pero podría ser de utilidad en el diagnóstico diferencial entre $\mathrm{HPB}$ y $\mathrm{CaP}$ cuando las biopsias sean dudosas, ya que el patrón de marcaje de VEGF de las células glandulares es diferente entre la células malignas y las hiperplásicas (Figuras 1 y 2).

Creemos que es necesario complementar este estudio con un marcaje adecuado de la microdensidad vascular, así como de las proteínas que se ven alteradas cuando las células malignas epiteliales adoptan un fenotipo mesenquimático que las hace capaces de desprenderse del epitelio, atravesar la lámina basal y migrar, degradando la matriz y modificando su citoesqueleto de modo de hacerse móviles permitiendo la generación de metástasis a distancia ${ }^{12}$. Sería interesante en estos pacientes estudiar el patrón de expresión de Ecadherina, beta-cateninas, vimentina, TWIST u otras proteínas que pudieran modificarse $\mathrm{e}^{6,26}$.

No se encontró diferencias en la magnitud de la presencia de VEGF en los pacientes que tuvieron una recidiva al año respecto a los pacientes que no la tuvieron, lo que nos indica que no tuvo utilidad como factor pronóstico para la recurrencia precoz del CaP en esta serie de pacientes. Dado que la recidiva se evaluó sólo un año posterior a la prostatectomía, no puede descartarse que en un seguimiento más prolongado de los enfermos, hubiera una correlación entre la magnitud de la tinción del VEGF y la recidiva bioquímica.

Otro resultado interesante de destacar es que no se encontró relación significativa entre el índice de Gleason de las biopsias y la recidiva al año. Lo mismo ocurrió con los valores de PSA preoperatorio. Esto nos indica que es necesario continuar con la búsqueda de factores pronósticos que sean útiles.

Una limitación de nuestro estudio fue la relativa falta de enmascaramiento en el proceso de observación y conteo de células marcadas, puesto que todos los observadores eran médicos o estudiantes de medicina con conocimiento en el área patológica. Los observadores no tuvieron acceso a los datos clínicos de los pacientes ni al informe de 
los patólogos, pero la arquitectura glandular en la mayoría de los casos probablemente fue sugerente.

En suma, encontramos una diferencia significativa en la marcación citoplasmática para VEGF entre biopsias de CaP y HPB. Esta podría ser una herramienta diagnóstica adicional en el análisis histopatológico de las biopsias dudosas. No hubo relación significativa entre el marcaje de VEGF y la presencia de recidiva al año; tampoco la hubo con ninguno de los otros factores pronósticos evaluados, como PSA preoperatorio e índice de Gleason.

Agradecimientos: a Jimena Leroy y a Makarena González por la colaboración con todos los procesos de laboratorio. Al Dr. Armando Cruzat, por su ayuda en el reclutamiento de pacientes.

\section{Referencias}

1. Departamento de Estadísticas e Información de Salud (DEIS). Tasa de Mortalidad por Tumores Malignos según Sexo, Chile 2000-2009. Consultado en http://deis. minsal.cl/vitales/defunciones_serie/Defunciones_Mortalidad_Tumores_Malignos_2000-2009.htm el día 1 de marzo de 2012.

2. Medina E, Kaempffer A. Mortalidad por cáncer en Chile: consideraciones epidemiológicas. Rev Med Chile 2001; 129: 1195-202.

3. O'Donnell H, Parker C. What is low-risk prostate cancer and what is its natural history? World J Urol 2008; 26: 415-22.

4. Sanda MG, Kaplan ID. A 64-year-old man with low-risk prostate cancer: review of prostate cancer treatment. JAMA 2009; 301: 2141-51.

5. Bermúdez H, Pizzi P, Notarantonio M, Avilés J. Experiencia en prostatectomía radical laparoscópica. Rev Chil Urol 2004; 69: 45-60.

6. Conacci-Sorrell M, Zhurinsky J, Ben-Ze'ev A. The cadherin-catenin adhesion system in signaling and cancer. J Clin Invest 2002; 109: 987-91.

7. González-Moreno O, Lecanda J, Green JE, Segura V, Catena R, Serrano D, et al. VEGF elicits epithelial-mesenchymal transition (EMT) in prostate intraepithelial neoplasia (PIN)-like cells via an autocrine loop. Exp Cell Res 2010; 316: 554-67.

8. Chen J, De S, Brainard J, Byzova TV. Metastatic properties of prostate cancer cells are controlled by VEGF. Cell Commun Adhes 2004; 11: 1-11.

9. Fidler IJ. Regulation of neoplastic angiogenesis. J Natl Cancer Inst Monogr 2001: 10-14.
10. Jackson MW, Roberts JS, Heckford SE, Ricciardelli C, Stahl J, Choong C, et al. A potential autocrine role for vascular endothelial growth factor in prostate cancer. Cancer Res 2002; 62: 854-9.

11. Melnyk O, Zimmerman M, Kim KJ, Shuman M. Neutralizing anti-vascular endothelial growth factor antibody inhibits further growth of established prostate cancer and metastases in a pre-clinical model. J Urol 1999; 161: 960-3.

12. Wallerand H, Robert G, Pasticier G, Ravaud A, Ballanger P, Reiter RE, et al. The epithelial-mesenchymal transition-inducing factor TWIST is an attractive target in advanced and/or metastatic bladder and prostate cancers. Urol Oncol 2010; 28: 473-9.

13. Stefanou D, Batistatou A, Kamina S, Arkoumani E, Papachristou DJ, Agnantis NJ. Expression of vascular endothelial growth factor (VEGF) and association with microvessel density in benign prostatic hyperplasia and prostate cancer. In Vivo 2004; 18: 155-60.

14. Klymkowsky MW, Savagner P. Epithelial-mesenchymal transition: a cancer researcher's conceptual friend and foe. Am J Pathol 2009; 174: 1588-93.

15. Kalluri R. EMT: when epithelial cells decide to become mesenchymal-like cells. J Clin Invest 2009; 119: 1417-9.

16. Ferrer FA, Miller LJ, Andrawis RI, Kurtzman SH, Albertsen PC, Laudone VP, et al. Vascular endothelial growth factor (VEGF) expression in human prostate cancer: in situ and in vitro expression of VEGF by human prostate cancer cells. J Urol 1997; 157: 2329-33.

17. Soulitzis N, Karyotis I, Delakas D, Spandidos DA. Expression analysis of peptide growth factors VEGF, FGF2, TGFB1, EGF and IGF1 in prostate cancer and benign prostatic hyperplasia. Int J Oncol 2006; 29: 305-14.

18. Wu TT, Wang JS, Jiaan BP, Yu CC, Tsai JY, Lin JT, Huang JK. Role of p21(WAF1) and p27(KIP1) in predicting biochemical recurrence for organ-confined prostate adenocarcinoma. J Chin Med Assoc 2007; 70: 11-5.

19. Walsh K, Sriprasad S, Hopster D, Codd J, Mulvin D. Distribution of vascular endothelial growth factor (VEGF) in prostate disease. Prostate Cancer Prostatic Dis 2002; 5: 119-22.

20. Acuña P, Ellwanger A, Ramírez A, Madrid E. Identificación de factor de crecimiento endotelial vascular (VEGF) en cáncer de próstata. Rev Chil Urol 2011; 76: 119-24.

21. Borre M, Nerstrom B, Overgaard J. Association between immunohistochemical expression of vascular endothelial growth factor (VEGF), VEGF-expressing neuroendocrine-differentiated tumor cells, and outcome in prostate cancer patients subjected to watchful waiting. Clin Cancer Res 2000; 6: 1882-90. 
Factor de crecimiento vascular endotelial (VEGF) en tejido prostático benigno y maligno - P. Acuña et al

22. Delongchamps NB, Peyromaure M. The role of vascular endothelial growth factor in kidney and prostate cancer. Can J Urol 2007; 14: 3669-77.

23. Peyromaure M, Badoual C, Camparo P, Grabar S, Goulvestre C, Fulla Y, et al. Plasma levels and expression of vascular endothelial growth factor-A in human localized prostate cancer. Oncol Rep 2007; 18: 145-9.

24. Peyromaure M, Camparo P, Badoual C, Descazeaud A, Dinh-Xuan AT. The expression of vascular endothelial growth factor is associated with the risk of cancer pro- gression after radical prostatectomy. BJU Int 2007; 99: 1150-3.

25. Mao K, Badoual C, Camparo P, Delongchamps NB, Vieillefond A, Dinh-Xuan AT, et al. The prognostic value of vascular endothelial growth factor (VEGF)-A and its receptor in clinically localized prostate cancer: a prospective evaluation in 100 patients undergoing radical prostatectomy. Can J Urol 2008; 15: 4257-62.

26. Klymkowsky MW. Beta-catenin and its regulatory network. Hum Pathol 2005; 36: 225-7. 